vereinzelte Untersuchungen vor. Zur Berechnung der Bandbreite wird ein Modell verwendet, welches auf dem mehrmaligen Durchgang eines Signals durch einen Verstärker mit frequenzabhängiger Verstärkung beruht. Die Bandbreite der Emission hängt dabei im wesentlichen von der Anfangsverstärkung und der Zahl der Durchgänge in der Anlaufphase ab (Zeitintervall zwischen Einsatz der Selbsterregung und merklicher Änderung der Inversion). Beim $Q$-Switch mittels Drehprisma ist die Anlaufphase kurz und die Bandbreite gross. Bei Verwendung eines sättigbaren Absorbers als $Q$-Switch dauert die Anlaufphase lange und die resultierende Bandbreite wird klein. Im Experiment konnte diese Abhängigkeit der Bandbreite vom Schaltertyp und von der Verstärkung bestätigt werden.

\title{
Ein Modell zur Entstehung von ultrakurzen Lichtpulsen aus dem Rauschen ${ }^{1}$ )
}

von E. MathieU und H. WeBer

(Institut für angewandte Physik, Universität Bern)

Gepulste Festkörperlaser, deren Emission durch passive, nichtlineare Absorber im Resonator gesteuert wird, liefern bei geeigneter Dimensionierung Lichtimpulse im Picosekundenbereich. Nach einem von Letokhov [1] stammenden Modell entwickeln sich diese Lichtimpulse aus dem Rauschen (spontane Emission) des Lasers, wobei der Absorber die Rauschimpulse nach der Pulshöhe diskriminiert (intensitätsabhängige Transmission) und in der Pulsdauer verringert. Der Laserverstärker hingegen bewirkt eine Verlängerung der Pulsdauer. Für diesen Prozess der Pulsentstehung wird ein einfaches Modell entwickelt, welches gestattet, den zeitlichen Verlauf von Pulsdauer und Spitzenintensität als Funktion von Absorber- und Verstärkereigenschaften zu berechnen. Ergebnisse werden angegeben.

\section{Literatur}

[1] V. S. Letoknov, Sov. Phys. JeTP 28, 562 (1969).

\section{Untersuchungen des spektralen Ausbleichens von Sättigungsabsorbern von R. WiLbrandt \\ (Institut für angewandte Physik, Universität Bern)}

Für den passiven $Q$-Switch von Festkörperlasern werden Lösungen organischer Farbstoffe verwendet. Das Absorptionsspektrum dieser sättigbaren Absorber kann auf homogener oder inhomogener Verbreiterung beruhen. Je nach Art seiner Verbreiterung beeinflusst der Absorber die Laseremission. Das Absorptionsverhalten des für Nd-Laser verwendeten Absorbers 9860 von Kodak wurde mit einer Ausbleichmethode untersucht. Es ergibt sich bei homogener Verbreiterung eine untere Grenze (30 A) für die homogene Breite oder bei inhomogener Verbreiterung eine obere Grenze (etwa $0,1 \mathrm{psec}$ ) für die Querrelaxationszeit.

1) Siehe ausführliche Publikation Z. angew. Math. Phys. 22, 458 (1971). 